OPEN ACCESS

Edited by:

Mingtao Zhao,

Nationwide Children's Hospital,

United States

Reviewed by:

Zhen Zhang,

Shanghai Children's Medical Center,

China

Kazuki Kodo,

Keio University, Japan

Yaozu Xiang,

Tongji University, China

${ }^{*}$ Correspondence:

Ning Sun

sunning@fudan.edu.cn

Chen Xu

xu_chen85@fudan.edu.cn

Qianqian Liang

qianqianliang@fudan.edu.cn

Specialty section:

This article was submitted to

Stem Cell Research,

a section of the journal

Frontiers in Cell and Developmental

Biology

Received: 07 April 2021

Accepted: 25 May 2021

Published: 17 June 2021

Citation:

Li B, Guo Y, Zhan Y, Zhou X, Li Y,

Zhao $C$, Sun $N, X u C$ and Liang $Q$

(2021) Cardiac Overexpression of XIN

Prevents Dilated Cardiomyopathy

Caused by TNNT2 $\triangle$ K210 Mutation.

Front. Cell Dev. Biol. 9:691749.

doi: 10.3389/fcell.2021.691749

\section{Cardiac Overexpression of XIN Prevents Dilated Cardiomyopathy Caused by TNNT2 $\Delta$ K210 Mutation}

\author{
Bin Li1,2, Yifan Guo', Yongkun Zhan' ${ }^{1}$ Xinyan Zhou', Yongbo Li', Chao Zhao ${ }^{3}$, \\ Ning Sun ${ }^{1,4 *}$, Chen $\mathrm{Xu}^{1 *}$ and Qianqian Liang ${ }^{1 *}$ \\ ${ }^{1}$ Department of Physiology and Pathophysiology, School of Basic Medical Sciences, Fudan University, Shanghai, China \\ 2 Shanghai Institute of Precision Medicine, Ninth People's Hospital, Shanghai Jiao Tong University School of Medicine, \\ Shanghai, China, ${ }^{3}$ Department of Medical Microbiology and Parasitology, School of Basic Medical Sciences, Fudan \\ University, Shanghai, China, ${ }^{4}$ Shanghai Key Laboratory of Clinical Geriatric Medicine, Institute of Integrative Medicine, Fudan \\ University, Shanghai, China
}

TNNT2 mutation is associated with a range of cardiac diseases, including dilated cardiomyopathy (DCM). However, the mechanisms underlying the development of DCM and heart failure remain incompletely understood. In the present study, we found the expression of cardiac XIN protein was reduced in TNNT2- $\mathrm{K} 210 \mathrm{hESCs-}$ derived cardiomyocytes and mouse heart tissues. We further investigated whether XIN protects against TNNT2 mutation-induced DCM. Overexpression of the repeatcontaining isoform XINB decreased the percentage of myofilaments disorganization and increased cell contractility of TNNT2- $\triangle \mathrm{K} 210$ cardiomyocytes. Moreover, overexpression of XINB by heart-specific delivery via AAV9 ameliorates DCM remodeling caused by TNNT2- $\triangle$ K210 mutation in mice, revealed by partially reversed cardiac dilation, systolic dysfunction and heart fibrosis. These results suggest that deficiency of XIN may play a critical role in the development of DCM. Consequently, our findings may provide a new mechanistic insight and represent a therapeutic target for the treatment of idiopathic DCM.

Keywords: dilated cardiomyopathy, gene therapy, TNNT2, cardiac remodeling, XIN

\section{INTRODUCTION}

Heart failure due to cardiomyopathy is among the most common causes of cardiovascular mortality (Harmon et al., 2005). Numbers of mutations in genes encoding sarcomeric proteins have been identified in individuals with cardiomyopathy (Morita et al., 2005; Villard et al., 2011). Cardiac troponin $\mathrm{T}$ (cTnT), encoded by TNNT2, is a cardiac muscle specific sarcomere protein that is important for the cell morphology, sarcomere assembly and myofilament contraction in cardiomyocytes (Solaro and Rarick, 1998; Wei and Jin, 2011). Deletion of lysine 210 ( $\Delta$ K210) in TNNT2 gene has been found to cause familial dilated cardiomyopathy (DCM) (Kamisago et al., 2000), which is characterized by dilated ventricular chamber and reduced systolic function leading to progressive heart failure with high mortality (Du et al., 2007; Kimura, 2010; Sfichi-Duke et al., 2010). Attempts to elucidate the molecular mechanisms underlying DCM mutations showed multiple molecular mechanisms including $\mathrm{Ca}^{2+}$ sensitivity of myofilament (Morimoto et al., 2002; Sfichi-Duke et al., 2010), remodeled intracellular calcium handling (Inoue et al., 2013; Lan et al., 2013) or interactions among the thin filaments protein constituents (Morimoto et al., 2002; 
Lombardi et al., 2008; Kimura, 2010) were involved in development of the disease. However, the detailed pathogenic mechanism underlying DCM disease remains poorly understood. Combined with human pluripotent stem cell (hPSC) technology and efficient cardiac differentiation protocols, human cardiomyocytes with corresponding gene mutations could be obtain in vitro, making it possible to expediently explore the effects of genetic mutations in the initial phases of disease development using isogenic human disease model systems (Sallam et al., 2014; Savla et al., 2014).

Xin actin-binding repeat-containing proteins (XIRPs) are a family of striated muscle-specific proteins defined by the presence of 15-28 copies of the conserved 16-aa XIN repeats. XIN is encoded by XIRP1 gene, also called hXina or CMYA1, is a striated muscle-specific gene and belongs to the XIRP family (Eulitz et al., 2013). Human XIRP1 gene gives rise to three XIN isoforms, known as XINA, XINB, and XINC (Molt et al., 2014). It is interesting that this gene has only been found in vertebrates with true chambered heart. XIN is expressed during early developmental stages of cardiac and skeletal muscles (Pacholsky et al., 2004). In cardiomyocytes, XIN is localized predominantly at intercalated discs (ICDs), which showed significance in the regulation of cardiac development and functions (Sinn et al., 2002). Multiple binding sites enabled XIN strengthen membrane apposition between cardiomyocytes by linking actin cytoskeleton to adherens junctions of ICDs (van der Ven et al., 2006; Wang et al., 2012, 2013). Additionally, its localization at adherens junctions of muscle cells and interaction with $\beta$-catenin, filaminC, and Mena/VASP imply a role in linking and transducing signals important for cardiac remodeling. Inactivation of Xin in chick embryos led to looping defects, abnormal beating behavior and edema (Wang et al., 1999), while Xin-deficient mice display myopathy, impaired contractility, and attenuated muscle repair (Al-Sajee et al., 2015), which indicating a crucial role in heart development and cardiac disease. Moreover, it has been observed that Xin may participate in a BMP-Nkx2.5-MEF2C pathway and promote the expression of ventricular myosin heavy chain (vMHC) gene to participate in cardiogenesis regulation (Wang et al., 1999, 2014). All these indicating an important role for XIN in early myofibrillogenesis and later in the maintenance of striated muscle integrity.

In the previous study, we characterized that the cellular phenotypes of TNNT2- $\triangle$ K210 hESC-CMs (human embroynic stem cells dervived cardiomyocytes) recapitulated DCM disease features (Li et al., 2021). Through whole transcriptomic analysis, down-regulated expression of XIN was identified in day35 TNNT2- $\triangle \mathrm{K} 210 \mathrm{hESC-CMs.} \mathrm{In} \mathrm{this} \mathrm{study,} \mathrm{we} \mathrm{further}$ confirmed the decreased expression of XIN in DCM models and focused on the therapeutic effects of XIN. We found that overexpression of XINB improved sarcomere organization and contraction force of TNNT2- $\triangle \mathrm{K} 210$ cardiomyocytes. In vivo cardiac-specific overexpression of XINB via AAV9 in neonatal TNNT2- $\triangle$ K210 DCM mice significantly rescued the DCM phenotypes. In conclusion, our findings uncovered the importance of XIN in TNNT2 mutation induced dilated cardiomyopathy.

\section{MATERIALS AND METHODS}

\section{Cell Culture and Cardiac Differentiation}

The human ESC line H7 obtained from WiCell Research Institute under specific Material Transfer Agreement was used in this study. All human stem cells researches followed the ISSCR Guidelines for the Conduct of Human Embryonic Stem Cell Research. Human ESCs were cultured using mTesR human pluripotent stem cell culture medium (STEMCELL Technologies) on Matrigel-coated (BD Biosciences) 6-well or 12well plates. Cardiac differentiation were performed according to previously described protocols (Lian et al., 2013). Each of three congenic lines were used in the following analysis. hESC lines between 70 and 80 passages were used in the following analysis.

\section{RNA Extraction and Real Time Quantitative PCR}

Total RNA was extracted using Trizol from Invitrogen according to the operating manual. The relative expression profiles of XIRP1 were examined by TOYOBO real-time PCR chemistry. Glyceraldehyde-3-phosphate dehydrogenase $(G A P D H)$ was used as endogenous control gene. PCR reaction were run in a final volume of $20 \mu \mathrm{l}$ composed of $1 \mu \mathrm{l}$ of cDNA, $9 \mu \mathrm{l}$ of buffer Mix and $100 \mathrm{nM}$ primer pairs. The PCR condition consisted of one initial denaturation at $95^{\circ} \mathrm{C}$ for $4 \mathrm{~min}$, followed by 40 cycles of denaturation at $95^{\circ} \mathrm{C}$ for $15 \mathrm{~s}$ and annealing at $60^{\circ} \mathrm{C}$ for $1 \mathrm{~min}$, which were performed by Bio-Rad iQ5 Real Time PCR System. All reactions were performed in triplicate. The primer sequences used are provided in Supplementary Table 1.

\section{Western Blot}

Cells or heart tissue were lysed with RIPA buffer $(50 \mathrm{mM}$ Tris $\mathrm{pH} 7.4,150 \mathrm{mM} \mathrm{NaCl}, 1 \%$ Triton X-100, 1\% sodium deoxycholate, $0.1 \%$ SDS) (Beyotime Biotechnology, P0013B) with protease inhibitor cocktail (APExBIO, K1012). Protein concentrations were determined by BCA Protein Assay Kit (TIANGEN, PA115). Equal amounts of protein were loaded on $10 \%$ SDS-PAGE. After electrophoresis, proteins were transferred to PVDF membrane (Millipore, IPVH00010). After blocked with 5\% skimmed milk at room temperature, membranes were incubated with primary antibodies (Xirp1: sc-166658, Santa Cruz Biotechnology, diluted at 1:1,000; GAPDH: 60,0041 -Ig, Proteintech, diluted at 1:1,000; $\beta$-tubulin: 10068-1-AP, Proteintech, diluted at $1: 2,000)$ at $4^{\circ} \mathrm{C}$ overnight and then incubated with corresponding secondary antibody. Western blots results were detected with Tanon 5200 Multi.

\section{Immunofluorescence Staining}

Morphological changes of hESC-cardiomyocytes were detected using immunostaining. After fixed with $4 \%$ paraformaldehyde and permeabilized with $0.05 \%$ Triton X-100, the cells were blocked with $4 \%$ goat serum, incubated with primary antibodies (cTnT: ab45932, Abcam, diluted at 1:400; Xirp1: sc-166658, Santa Cruz Biotechnology, diluted at $1: 100$ ) overnight at $4^{\circ} \mathrm{C}$, and then visualized with secondary antibody. Frozen left ventricular tissue slides were incubated with Alexa Fluor ${ }^{\text {TM }}$ 
488 Conjugated-Wheat Germ Agglutinin (Life Technologies, United States) and DAPI (Life Technologies) to visualize myocyte membranes. Fluorescence images were obtained with a confocal microscope (LEICA SP8).

\section{Echocardiography}

Echocardiography was performed at determined time. Mice were anesthetized by $1.5 \%$ isoflurane and M-mode images were captured and measured using the small animal echocardiography analysis system at the midventricular level. The echocardiographer was blinded to mouse genotypes or treatments.

\section{Histological Analysis}

Cardiac tissue from mice at 3 months of age was fixed in $4 \%$ formaldehyde and paraffin embedded. The sections were sliced at $5 \mu \mathrm{m}$. Hematoxylin-eosin (H\&E) and Masson staining were performed to examine general histology and analyze cardiac fibrosis, respectively. The sections were observed and photographed by the microscope. For the detection of transmission electronmicroscopy, heart tissues from ventricle were immediately fixed in $2.5 \%$ glutaraldehyde solution for about $1 \mathrm{~h}$ at room temperature and post-fixed for $3 \mathrm{~h}$. Samples were embedded, sectioned at about $70 \mathrm{~nm}$ thickness, and stained with lead citrate for $8 \mathrm{~min}$. Micrographs were captured using a PHILIPS CM-120 transmission electron microscope.

\section{Lentivirus Production}

$\mathrm{XINB}$ cDNA was cloned into target gene lentiviral vector ( $\mathrm{pCDH}$ EF1-MCS-T2A-copGFP) at the MCS locus. The HEK293T cells were transfected with the target gene lentiviral vector plasmid and packaging plasmids using Lipofectamine 3,000 (Life Technologies). The transfected cells were cultivated and collected supernatant every $24 \mathrm{~h}$. Ultrafiltration was used to concentrate the medium. Lentiviruses expressing EGFP gene were used as the control. The efficiency of virus transduction was confirmed $24 \mathrm{~h}$ after infection.

\section{Recombinant AAV9 Virus Production}

We cloned XINB or Luciferase cDNA into ITR-containing AAV9 plasmid with cardiac specific TNNT2 promoter and obtained the pAAV:TNNT2:XINB and pAAV:TNNT2:Luciferase plasmids. AAV9 was packaged using HEK293 cells with AAV9:Rep-Cap and pHelper and purified by an iodixanol gradient centrifugation. AAV9 titer was determined by quantitative PCR. $50 \mu l$ containing $1 \times 10^{12}$ vg AAV9-Luci or AAV9-XINB virus were used for one mouse. Intraperitoneal injection of AAV9 was performed within 3 days of birth.

\section{Contraction Force Measurements}

HESC-cardiomyocytes were separated into single cell and cultured on a $0.4-0.8 \mathrm{~mm}$ thick mattress of undiluted Matrigel for 2-3 days. We used video-based edge detection to measure contractility, which were visualized using a Zeiss CFM-500 inversion fluorescence microscope. Spontaneous contraction traces were recorded and analyzed by FelixGX software (PTI).

\section{In vivo Bioluminescence Imaging}

In vivo bioluminescence imaging was performed to confirm AAV-9 mediated gene expression. $15 \mathrm{~min}$ after intraperitoneal injection of D-luciferin (Promega, $15 \mathrm{mg} / \mathrm{ml}, 0.2 \mathrm{ml}$ ) under anesthesia with inhalation of isoflurane, the mice were placed for imaging using an IVIS Lumina K system (PerkinElmer).

\section{Animals}

A knock-in mouse with deletion of Lys-210 mutation in cardiac troponin T gene (TNNT2- $\Delta \mathrm{K} 210$ ) was used as the DCM model animal, which were constructed by VIEWSOLID biotechnology co., LTD. All procedures involving animal use, housing, and operations in this study were approved by the Institutional Animal Care and Use Committee of the Fudan University and met the Guide for the Care and Use of Laboratory Animals (National Institutes of Health).

\section{Statistical Analysis}

All data are presented as mean \pm SEM (standard error of the mean). Two normally distributed data sets analysis was performed using unpaired two-tailed Student's $t$-test. Oneway ANOVA was used to test for significant differences in multiple group comparisons. $P<0.05$ was considered statistically significant. ${ }^{*} P<0.05,{ }^{* *} P<0.01,{ }^{* * *} P<0.001$.

\section{RESULTS}

\section{XIN Deficiency Was Detected in the TNNT2- $\triangle$ K210 hESC-Derived Cardiomyocytes}

HESCs (H7) lines with either a DCM-causing heterozygous $\Delta \mathrm{K} 210(\Delta \mathrm{K} 210 / \mathrm{WT})$ or homozygous $\Delta \mathrm{K} 210(\Delta \mathrm{K} 210 / \Delta \mathrm{K} 210)$ mutation in the TNNT2 gene were generated as described before (Li et al., 2021). Through modification of the WNT signaling pathway, we differentiated the isogenic heterozygous and homozygous TNNT2- $\triangle \mathrm{K} 210$ mutant hESCs into cardiomyocytes and beating cells were observed on day $8 \sim 9$ after cardiac differentiation (Figure 1A). Immunostaining of WT, and TNNT2- $\triangle \mathrm{K} 210$ hESCs-derived cardiomyocytes showed positive staining of cardiac markers cTnT, $\alpha$-actinin and MLC2V (Supplementary Figure 1A). Fluorescent activated cell sorting (FACS) analyses for cTnT showed a 90\% purity of cTnT + cardiomyocytes in our cardiac differentiation and similar efficiencies among H7 WT and TNNT2- $\triangle$ K210 hESC lines (Supplementary Figure 1B). In the previous study, we characterized that the cellular phenotypes, such as $\mathrm{Ca}^{2+}$ handling, and contractility of the TNNT2- $\triangle \mathrm{K} 210$ cardiomyocytes recapitulated DCM disease features ( $\mathrm{Li}$ et al., 2021). We further analyzed whole transcriptomic results of day35 WT (WT/WT) and TNNT2- $\Delta$ K210 cardiomyocytes (Supplementary Figure 2A). Since there are no reports of patients carrying homozygous TNNT2- $\Delta \mathrm{K} 210$ mutation, heterozygous TNNT2- $\triangle \mathrm{K} 210$ cardiomyocytes may have a stronger disease-related significance. Through comparing with WT cardiomyocytes and RT-qPCR analysis, we found 


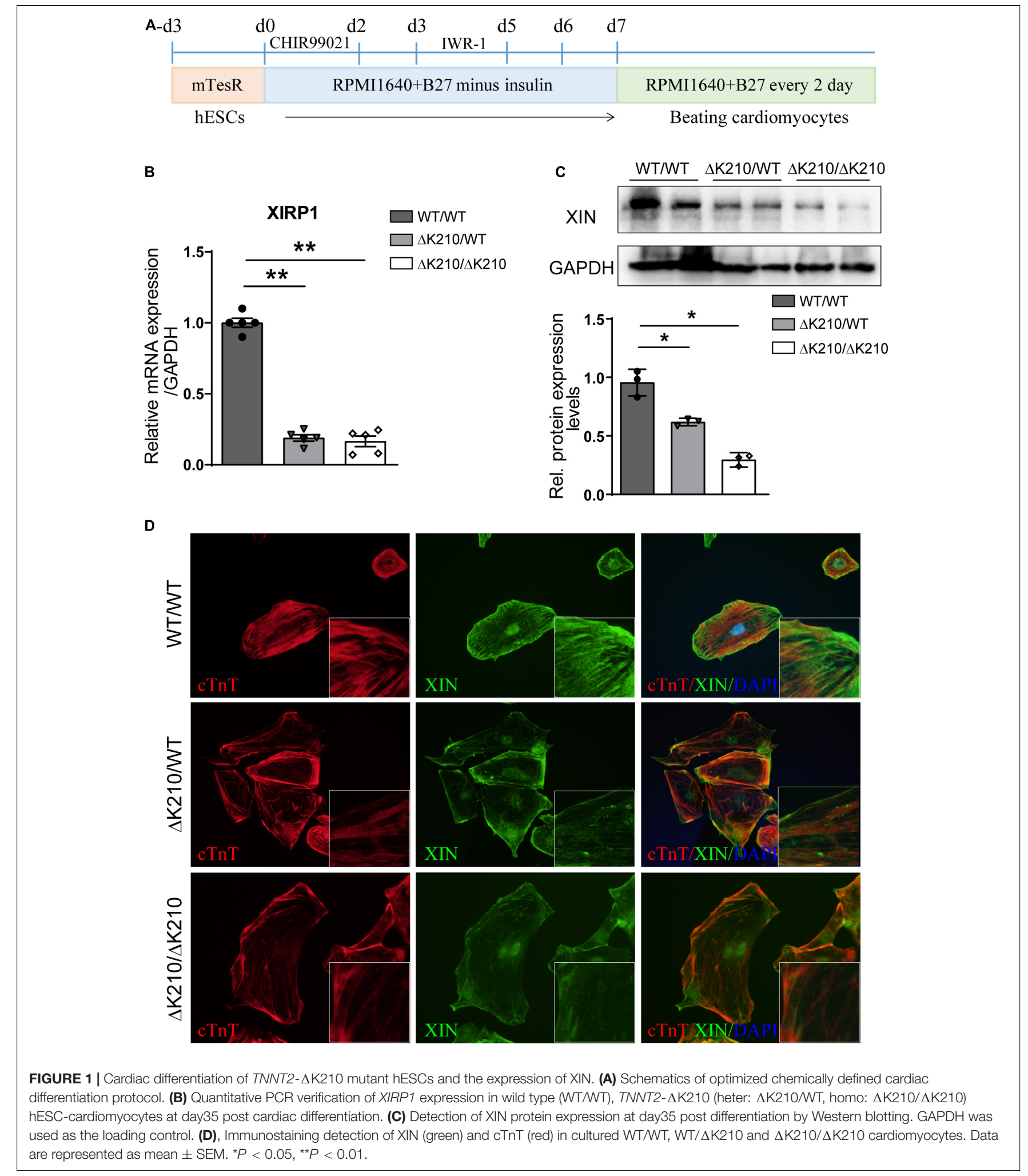

XIRP1 was sharply down-regulated and the encoded XIN also decreased in both heterozygous and homozygous TNNT2$\Delta$ K210 cardiomyocytes at day35 post cardiac differentiation (Figures 1B,C and Supplementary Figures 2B,C). Previous studies showed XIN is an F-actin-binding protein and expressed in the early stages of development of cardiac muscles, so we hypothesized that reduction of XIN participated in the development of dilated cardiomyopathy. Localization and 
A

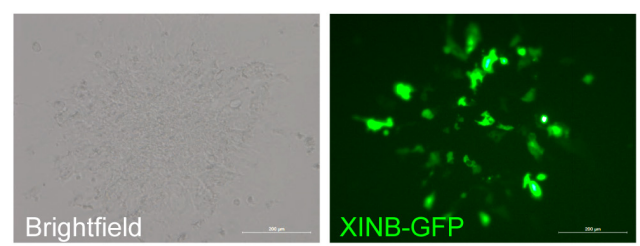

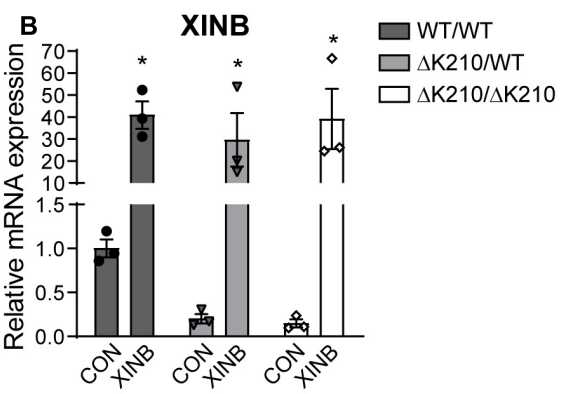

C
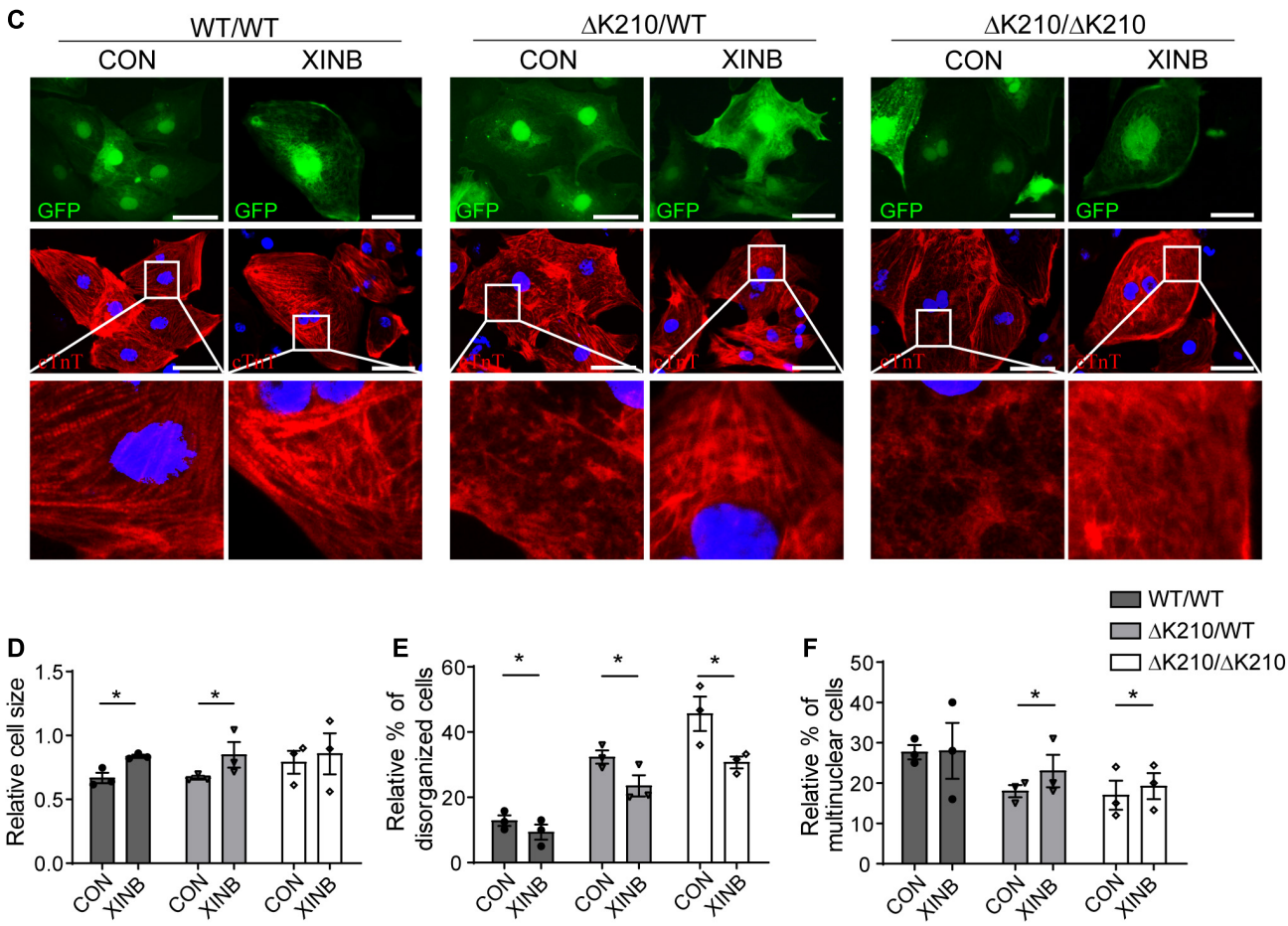

FIGURE 2 | Overexpression of XINB partially rescued the DCM phenotype of TNNT2- $\triangle$ K210 cardiomyocytes. (A) Brightfield and fluorescence images of hESC-cardiomyocytes illustrating green fluorescence protein (GFP) expression after XINB-lentivirus transfection. Scale bars, 200 mm. (B) Relative XINB expression in WTMT, WT/ $\mathrm{K} 210$, and $\Delta \mathrm{K} 210 / \Delta \mathrm{K} 210$ cardiomyocytes determined by quantitative PCR. (C) Representative immunostaining of cTnT (red) in cultured WT/WT, $\mathrm{WT} / \Delta \mathrm{K} 210$, and $\Delta \mathrm{K} 210 / \Delta \mathrm{K} 210$ cardiomyocytes after XINB overexpression. Cells transfected with lentivirus expressing GFP were used as control. Scale bars, 50 $\mathrm{mm}$. (D) Quantification of relative cell size and (E) statistics of the relative disorganized sarcomeric pattern for WTMT, WT/ $\Delta$ K210, and $\Delta K 210 / \Delta K 210$ cardiomyocytes after overexpression of XINB. (F) Percent of multinuclear cells increased after XINB overexpression in TNNT2- $\Delta$ K210 cardiomyocytes. $n>30,3$ lines in each group; data are represented as mean \pm SEM. ${ }^{\star} P<0.05$.

myofilament pattern of XIN in WT and TNNT2- $\triangle \mathrm{K} 210$ cardiomyocytes were then further examined. Compared to WT cardiomyocytes, staining patterns of XIN combined with myofilaments cTnT were changed and weaker in TNNT2- $\triangle \mathrm{K} 210$ cardiomyocytes (Figure 1D). These results suggested a possible role of XIN in the regulation of cardiomyocytes remodeling.

\section{XINB Improved Myofilaments Organization in TNNT2- $\Delta$ K210 Cardiomyocytes}

Because of the limitations of lentivirus and recombinant adenoassociated virus vector capacity, we used isoform of XINB (containing XIN-repeats, peptide motifs which bind actin filaments by coiling around them) in the next in vitro and in vivo study. We overexpressed XINB in TNNT2- $\triangle \mathrm{K} 210$ cardiomyocytes to check whether restore of XINB can rescue the DCM-like cellular phenotypes. Lentiviral vectors containing XINB-T2A-GFP were generated, with GFP cassette to indicate the transduced cells, while GFP only was used as the negative control. Cardiomyocytes were infected with XINB or control lentiviruses for 7 days. Obvious GFP (Figure 2A) and increased XINB (Figure 2B) were detected after transfection. As XIN binding to actin filaments and may play an important role in early myofibrillogenesis, we then used immunofluorescence to detect the myofilament structure after XINB overexpression. Compared to WT cardiomyocytes, myofibrils were poorly organized with diffusions and damages (myofilament staining 
was weaker and less dense, punctate distribution of sarcomeric cTnT over one fourth of the total cellular area) (Sun et al., 2012) in $\Delta \mathrm{K} 210$ cardiomyocytes. While XINB overexpressed $\Delta \mathrm{K} 210$ cardiomyocytes showed better organized and dense myofilaments over the entire cellular area (Figure 2C). An increase in cell size of WT/WT and $\Delta$ K210/WT cardiomyocytes and a reduced percentage of TNNT2- $\Delta \mathrm{K} 210$ cardiomyocytes with disorganized cTnT distribution patterns were observed after XINB expression (Figures 2D,E). We also found higher frequency of multinucleation in XINB overexpressed TNNT2$\Delta \mathrm{K} 210$ cardiomyocytes versus control (Figure $2 \mathrm{~F}$ ), which might suggest a further mature state. In conclusion, phenotypes of TNNT2- $\triangle \mathrm{K} 210$ cardiomyocytes such as dispersion and disorganization of myofilaments were improved after XINB overexpression. These results showed that XIN may play an important role in myofibrillar assembly and the maintenance of muscle integrity.

\section{Overexpression of XINB Increased Contractile Force of hESC-Derived Cardiomyocytes}

To further evaluate the effects of XINB overexpression, we next examined the contractile force of cardiomyocytes. Compared with control, XINB treated WT/WT, $\Delta$ K210/WT and $\Delta \mathrm{K} 210 / \Delta \mathrm{K} 210$ cardiomyocytes showed increased contractility (Figures 3A-D). It was previously reported that, in chicken, cXin participated in a BMP2-Nkx2.5-Mef2C-cXin-vMHC pathway to regulate cardiogenesis (Wang et al., 2014). Then we further examined expression of genes such as TNNT2, ACTN2 and MYH7, which related to cardiac muscle contraction, and found they were also up-regulated after XINB overexpression (Figures 3E-G), suggesting an improvement in cardiomyocyte contractile function. And the increased expression of cardiac structural genes after XINB transfection may also contribute to the enlarged cell size, improved myofilaments organization and maturation of cardiomyocytes in Figures 2D-F.

\section{XIN Was Down-Regulated in the TNNT2- $\Delta$ K210 Mice Hearts}

We next detected the expression of Xin in heart tissues of wild type and TNNT2- $\triangle$ K210 mice at different days after birth. The results indicated that Xirp1 expression was also down-regulated in the TNNT2- $\triangle \mathrm{K} 210$ mice since they were born (Figure 4A). Western blot analysis showed decreased level of XIN (encoded by Xirp1) protein in TNNT2- $\Delta \mathrm{K} 210$ mice hearts 7 days after birth (Figures 4B,C).

\section{XINB Protected Against Dilated Cardiomyopathy in TNNT2- $\Delta$ K210 Mice}

To assess whether XINB could rescue the phenotype of DCM in vivo, we next used recombinant adeno-associated virus type 9 (AAV9) to specifically express XINB in the heart of neonatal TNNT2- $\triangle \mathrm{K} 210$ DCM mice (Figure 5A). Plasmid AAV9: TNNT2: XINB (AAV9-XINB) with XINB driven by TNNT2 promoter was generated to express XINB specificially in cardiomyocytes, while AAV9: TNNT2: Luciferase (AAV9Luci), in which XINB was replaced by luciferase, was used as control. To validate the efficiency of viral delivery, uninjected mice were used as controls. The luciferase protein was detected in hearts but not in any other organs of the AAV9-Luci treated mice, and also not found in the uninjected mice (Figure 5B). Functions of the wild type and TNNT2- $\Delta$ K210 transgenic mice were assessed using echocardiography at 1 and 3 months of age. The representative $\mathrm{M}$-mode echocardiographic images at 3 months of age were shown in Figure 5C. LV ejection fraction (Figure 5D) and fractional shortening (Figure 5E) of the XINBtreated TNNT2- $\triangle \mathrm{K} 210$ mice were significantly increased by $64.3 \pm 3.7 \%$ to $78.6 \pm 3.1 \%$ and $28.8 \pm 3.1 \%$ to $40.0 \pm 2.8 \%$ compared with control. The left ventricular (LV) end systolic and diastolic diameter of the mice was decreased by $2.5 \pm 0.2 \mathrm{~mm}$ to $2.0 \pm 0.06 \mathrm{~mm}$ and $4.3 \pm 0.2 \mathrm{~mm}$ to $3.8 \pm 0.1 \mathrm{~mm}$ at 3 months of age compared with that of the AAV9-luciferase injected TNNT2- $\triangle \mathrm{K} 210$ transgenic mice (Figures 5F,G). These results suggested that the cardiac function of TNNT2- $\triangle \mathrm{K} 210$ mice was significantly improved after XINB overexpression.

\section{XINB Improved Cardiac Pathological Phenotypes in the TNNT2- $\Delta$ K210 Mice}

The morphological changes of heart ventricles and cardiomyocytes were further determined by histological examination (Figures 6A-E). Overexpression of XINB in the TNNT2- $\triangle \mathrm{K} 210$ transgenic mice significantly reduced the ventricular dilatation and accumulation of collagen in the interstitial space compared with the typical histological changes observed in the heart tissues of the control TNNT2- $\Delta \mathrm{K} 210$ mice (Figures 6A,B). At 3 months of age, TNNT2- $\Delta \mathrm{K} 210$ mice also showed cardiomyocytes enlargement compared to WT mice. XINB overexpression prevented the development of heart dilation and hence recovered the size of myocytes (Figures 6C,D). Twisted Z-line, sparse myofilaments, and swollen mitochondria was also seen in the control TNNT2$\Delta \mathrm{K} 210$ mice hearts. However, the disrupted sarcomeric structures of heart was improved by XINB overexpression, which showed relative dense and aligned myofilaments with mitochondria arranged in the gap (Figure 6E). These changes indicated that the morphological phenotypes of DCM in TNNT2$\Delta \mathrm{K} 210$ mutant mice were partially rescued by cardiac-specific expression of XINB.

\section{DISCUSSION}

Detailed mechanisms for dilated cardiomyopathy caused by TNNT2 gene mutation remain largely unknown. Current research using dilated myocardium from patients at a relative late disease stage might neglect the earliest molecular events which is key for DCM development. In this study, we used cardiomyocytes derived from hESCs which carried the DCMcausing TNNT2- $\triangle$ K210 mutation and confirmed XIN decreased compared with control. Furthermore, XINwas also decreased in DCM mouse heart. Overexpression of the repeat-containing isoform XINB in $\Delta \mathrm{K} 210$ cardiomyocytes improved myofilaments 

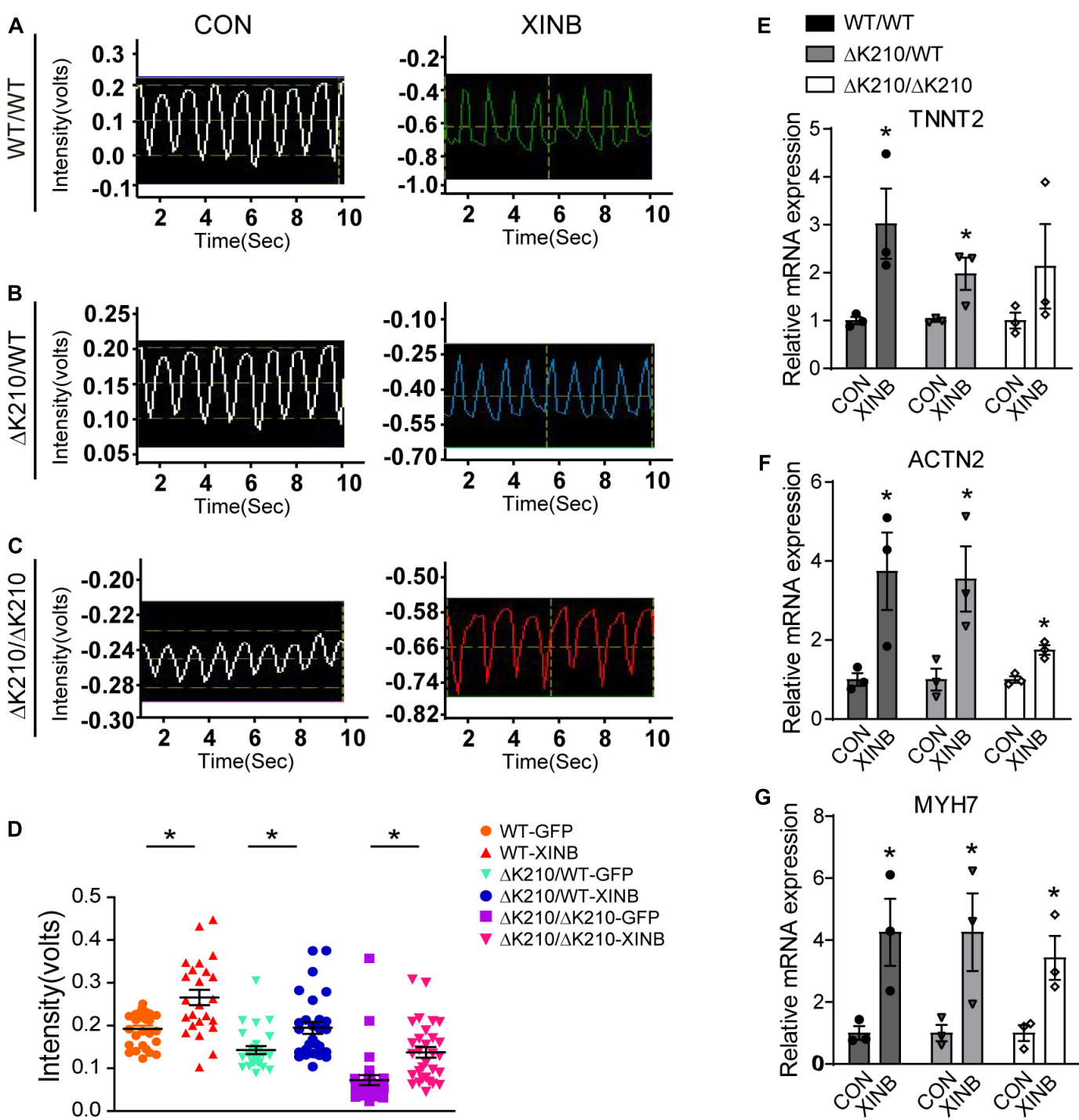

- WT-GFP

- WT-XINB

$\triangle$ K210/WT-GFP

- $\triangle \mathrm{K} 210 \mathrm{NT}-\mathrm{XINB}$

$\Delta K 210 / \Delta K 210-G F P$

$\nabla \Delta K 210 / \Delta K 210-X I N B$

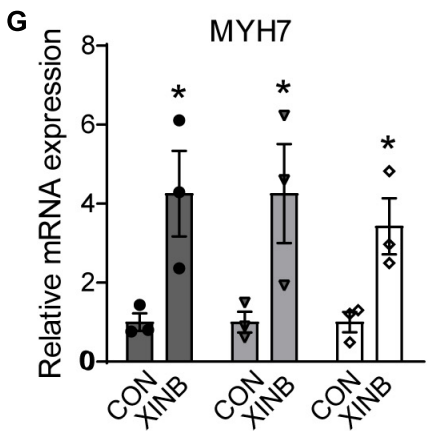

FIGURE 3 | XINB increased contractility of TNNT2- $\triangle$ K210 cardiomyocytes. (A) Representative video recording traces of spontaneous contraction of single WTMT. (B) $\Delta \mathrm{K} 210 / \mathrm{WT}$ and (C) $\Delta \mathrm{K} 210 / \Delta \mathrm{K} 210$ cardiomyocytes in control or XINB overexpression group. (D) Statistics of video recording intensities showed increased contractile forces after XINB overexpression in WTMT $(n=29), \Delta \mathrm{K} 210 / \mathrm{WT}(n=30)$, and $\Delta \mathrm{K} 210 / \Delta \mathrm{K} 210(n=30)$ cardiomyocytes from 3 independent lines in each group. Relative expression of cardiac muscle contraction related genes (E) TNNT2, (F) ACTN2, and (G) MYH7 increased after XINB overexpression. Data are represented as mean \pm SEM. * $P<0.05$.
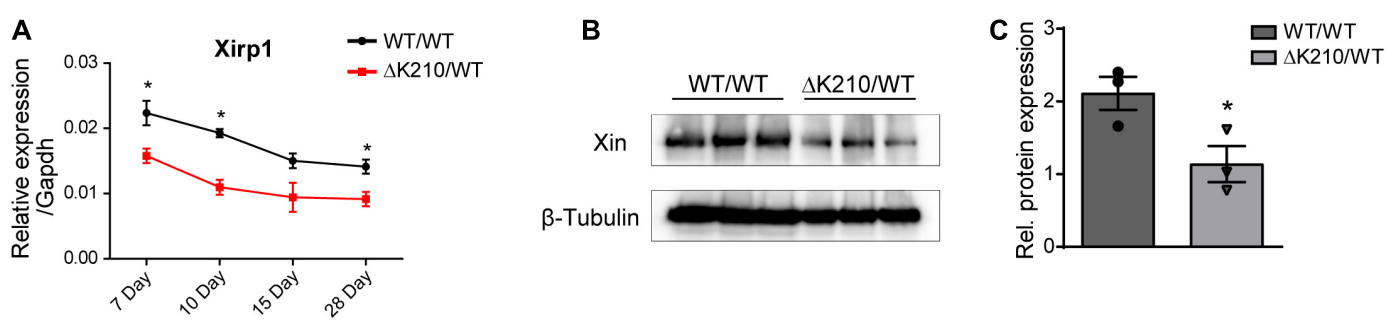

FIGURE 4 | Expression of Xirp1 in the TNNT2- $\Delta$ K210 mice hearts. (A) Changes in temporal mouse Xirp1 expression in wild type and TNNT2- $\Delta$ K210 mutant mice. (B) Western blot analysis showed decreased level of XINprotein in Day 7 TNNT2- $\Delta$ K210 mice hearts. (C), Quantification of western blot signals in (B) and normalized to the loading control ( $\beta$-Tubulin). Data are represented as mean \pm SEM. ${ }^{\star} P<0.05$. 
A

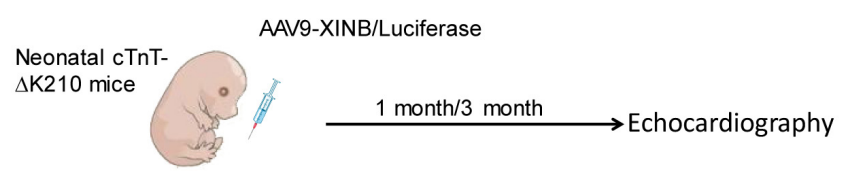

B

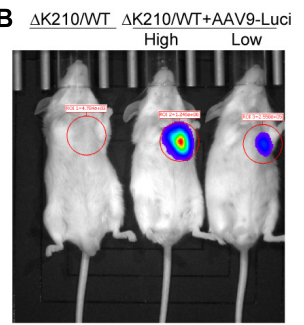

C
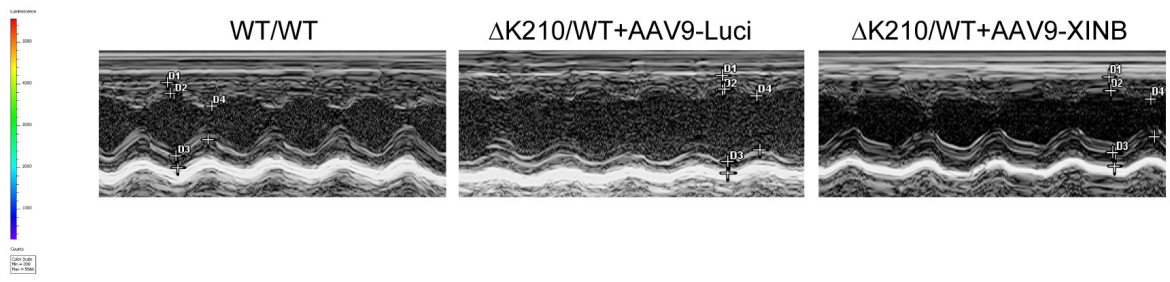

ind
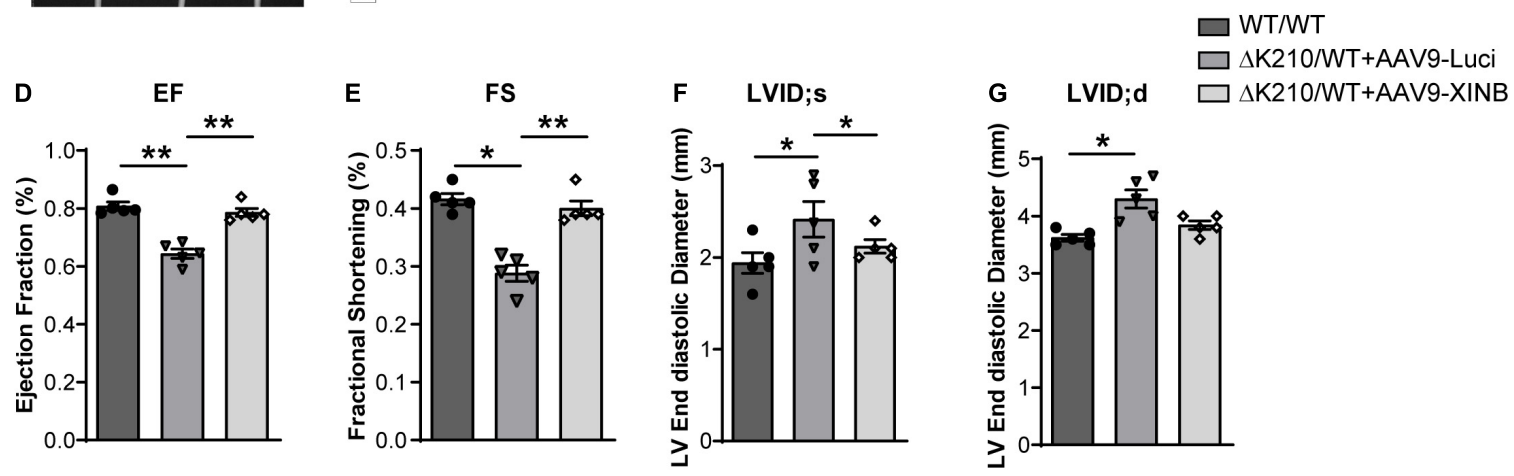

FIGURE 5 | Cardiac-specific expression of XINB improved cardiac function of TNNT2- $\triangle$ K210 mice. (A) Schematic illustration of the design of XINB delivery through AAV9. (B) In vivo bioluminescence imaging of mice tested the efficiencies of AAV9-Luci with the cTnT promoter for cardiomyocyte-specific gene expression. 3-day-old neonatal mice were intraperitoneally injected with AAV9-Luci viruses ( $1 \times 10^{12}$ viral genomes per mouse). Mouses without AAV injection were used as control. High: high dose. Low: low dose. (C) Representative M-mode echocardiography recordings of 3-months-old WT mice, AAV9-Luci injected TNNT2- $\mathrm{K}$ K210 mice, and AAV9-XINB injected TNNT2- $\triangle$ K210 mice. (D) Echocardiographic analysis of ejection fraction (EF), (E) fractional shortening (FS), (F) left ventricular end systolic internal diameter (LVID; s), and (G) left ventricular end diastolic internal diameter (LVID; d) of TNNT2- $\Delta$ K210 mice after XINB treatment ( $n=5$ per group). Data are represented as mean \pm SEM. ${ }^{*} P<0.05,{ }^{* *} P<0.01$.

organization and increased cell contractile force. Increase in the LVEF and LVFS after XINB treatment via AAV9 indicated that overexpression of XINB also improved heart functions in vivo. These results indicated XIN deficiency might be involved in the resulted loosely organized myofilament and systolic dysfunction caused by the TNNT2- $\Delta \mathrm{K} 210$ mutation and overexpression of XINB could prevent DCM cardiac remodeling caused by the mutation.

Intra-exonic splicing of XIRP1 gene resulted in three XIN isoforms: XINA (a full length protein), XINB (lacks filamin C-binding carboxy terminus) and truncated XINC that lacks XIN repeats, $\beta$-catenin-binding region and most of functional regions (van der Ven et al., 2006). Because of the limitations of lentivirus or recombinant adeno-associated virus vector capacity, we used XINB in this study. And we speculate XINA, as a full length protein, will work as well as XINB. Previous study revealed XINC protein only existed in hypertrophic tissues by comparing normal and hypertrophic human cardiac samples (Otten et al., 2010), suggesting that this isoform might also be involved in the pathogenesis of cardiomyopathy. Organization of individual components into thick or thin filaments and their integration into M-bands and Z-lines are important for myofibrillar assembly (Claeys et al., 2009; Sanger et al., 2010).
Consistent with our results, survey of microarray datasets in Gene Expression Omnibus (GEO) has revealed that XIRP1 downregulated in failing hearts from human patients with diabetic or non-diabetic heart failure (GDS4314), and idiopathic DCM (GDS651) (Wang et al., 2014). XIN repeats as an actin-binding motif, directly bind actin filaments in vitro and cross link these filaments into networks in a similar manner as nebulin repeats. Within the XIN repeats, the overlapped $\beta$-catenin-binding domain, Ena/VASP binding domain DNAbinding domain and proline-rich region were identified from human XIN (van der Ven et al., 2006; Wang et al., 2012, 2013). XIN act as part of a complex that participate in the early morphogenesis of the heart and the maintenance of striated muscle integrity (Claeys et al., 2009). Deficiency of XIN might disrupt cytoskeletal protein arrangements and interactions, further weaken the stability of myofibril assembly and maintenance, thus leads to DCM.

In the present study, we were able to successfully rescue cardiac remodeling in a TNNT2- $\triangle \mathrm{K} 210$ mouse model of dilated cardiomyopathy by overexpressing XIN. XIN deficiency might be a key mechanism of TNNT2- $\triangle \mathrm{K} 210$ mutation-caused familial DCM and XIN could act as an interventional target for the relevant heart diseases. 

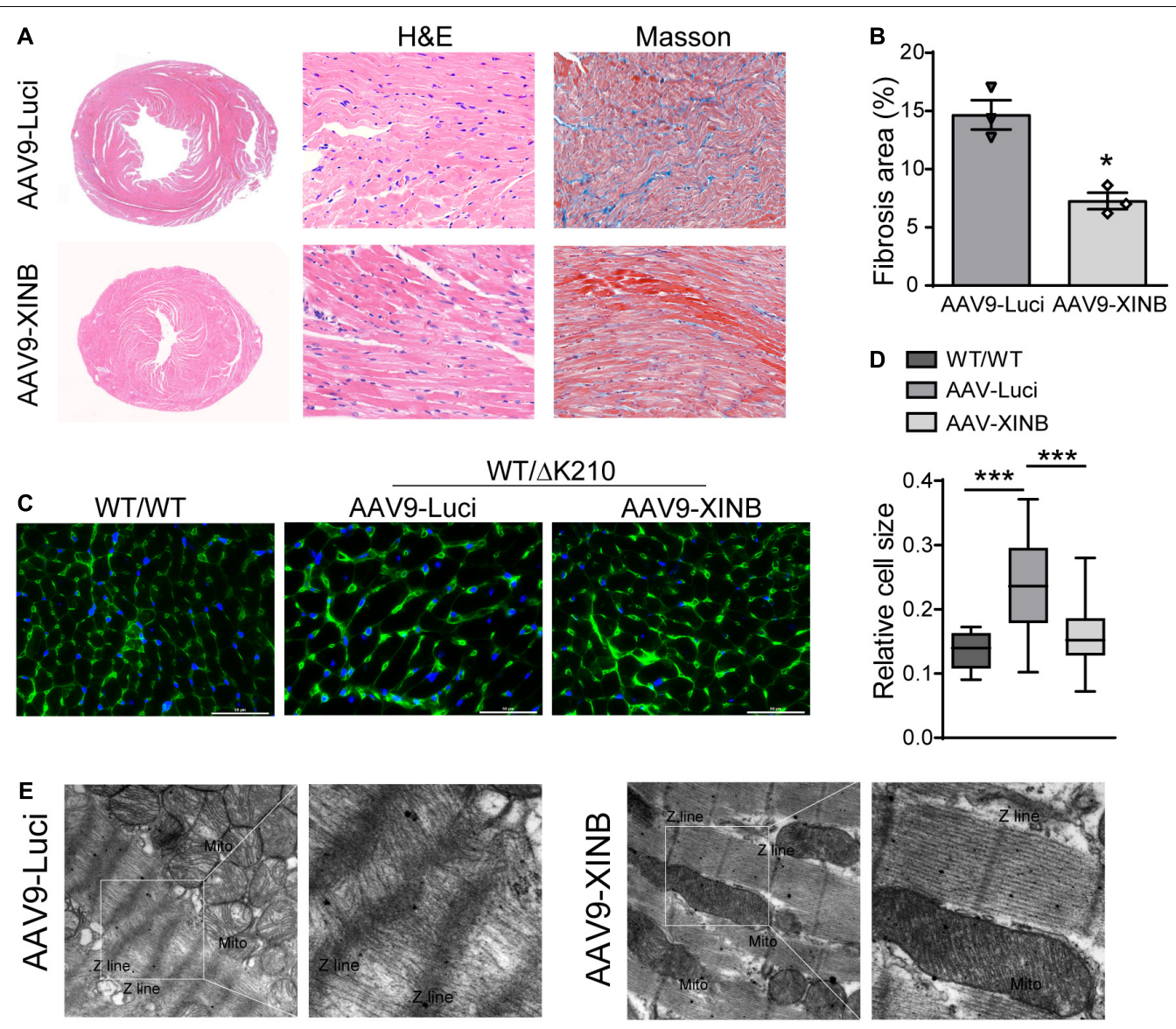

FIGURE 6 | Morphological studies showed cardiac-specific expression of XINB in TNNT2- $\Delta$ K210 mice rescued DCM phenotypes. (A), Representative H\&E staining of the hearts from 3-months old control and AAV9-XINB injected TNNT2- $\triangle$ K210 mice. Masson's trichrome staining showed less fibrosis in the mutant LV tissue when compared to control. (B) Quantification of interstitial fibrosis area in each group. (C,D) Wheat germ agglutinin (WGA; as an indicator of cardiomyocyte size) staining of left ventricle sections and quantification of myocyte sizes. Scale bars, $50 \mathrm{~mm}$. (E) Ultrastructure of the left ventricular tissue sections from 3-months old mice revealed by transmission electron microscopy at $\times 6,000$ magnification. AAV9-XINB treatment significantly improved the sarcomeric structure of TNNT2- $\triangle$ K210 mice. Data are represented as mean \pm SEM. ${ }^{*} P<0.05$, ${ }^{* * *} P<0.001$.

\section{DATA AVAILABILITY STATEMENT}

The original contributions presented in the study are included in the article/Supplementary Material, further inquiries can be directed to the corresponding author/s.

\section{ETHICS STATEMENT}

The animal study was reviewed and approved by Institutional Animal Care and Use Committee of the Fudan University.

\section{AUTHOR CONTRIBUTIONS}

$\mathrm{BL}, \mathrm{NS}, \mathrm{QL}$, and CX conceived the research and wrote the manuscript. BL, YG, YZ, XZ, YL, and $\mathrm{CZ}$ performed the experiments and data analysis. All authors contributed to the article and approved the submitted version.

\section{FUNDING}

This work was supported by the National Natural Science Foundation of China (NSFC No. 82070391) to NS, the National Key R\&D Program of China (No. 2018YFC2000202) to NS, the Postdoctoral Science Foundation (No. KLH1322109) to BL, the Young Elite Scientist Sponsorship Program by CAST (No. 2018QNRC001) to QL, the funding of Innovative research team of high-level local universities in Shanghai and a key laboratory program of the Education Commission of Shanghai Municipality (No. ZDSYS14005) to QL and CX, and the National Key Research and Development Program of China (No. 2018YFC2000500/03) to $\mathrm{CZ}$.

\section{SUPPLEMENTARY MATERIAL}

The Supplementary Material for this article can be found online at: https://www.frontiersin.org/articles/10.3389/fcell.2021. 691749/full\#supplementary-material 


\section{REFERENCES}

Al-Sajee, D., Nissar, A. A., Coleman, S. K., Rebalka, I. A., Chiang, A., Wathra, R., et al. (2015). Xin-deficient mice display myopathy, impaired contractility, attenuated muscle repair and altered satellite cell functionality. Acta Physiol. 214, 248-260. doi: 10.1111/apha.12455

Claeys, K. G., Van Der Ven, P. F., Behin, A., Stojkovic, T., Eymard, B., Dubourg, O., et al. (2009). Differential involvement of sarcomeric proteins in myofibrillar myopathies: a morphological and immunohistochemical study. Acta Neuropathol. 117, 293-307. doi: 10.1007/s00401-008-0479-7

Du, C. K., Morimoto, S., Nishii, K., Minakami, R., Ohta, M., Tadano, N., et al. (2007). Knock-in mouse model of dilated cardiomyopathy caused by troponin mutation. Circ. Res. 101, 185-194. doi: 10.1161/circresaha.106.14 6670

Eulitz, S., Sauer, F., Pelissier, M. C., Boisguerin, P., Molt, S., Schuld, J., et al. (2013). Identification of Xin-repeat proteins as novel ligands of the $\mathrm{SH} 3$ domains of nebulin and nebulette and analysis of their interaction during myofibril formation and remodeling. Mol. Biol. Cell. 24, 3215-3226. doi: 10.1091/mbc. e13-04-0202

Harmon, W. E., Mcdonald, R. A., Reyes, J. D., Bridges, N. D., Sweet, S. C., Sommers, C. M., et al. (2005). Pediatric transplantation, 1994-2003. Am. J. Transplant. 5, 887-903.

Inoue, T., Kobirumaki-Shimozawa, F., Kagemoto, T., Fujii, T., Terui, T., Kusakari, Y., et al. (2013). Depressed Frank-Starling mechanism in the left ventricular muscle of the knock-in mouse model of dilated cardiomyopathy with troponin T deletion mutation DeltaK210. J. Mol. Cell. Cardiol. 63, 69-78. doi: 10.1016/j. yjmcc.2013.07.001

Kamisago, M., Sharma, S. D., Depalma, S. R., Solomon, S., Sharma, P., Mcdonough, B., et al. (2000). Mutations in sarcomere protein genes as a cause of dilated cardiomyopathy. N. Engl. J. Med. 343, 1688-1696. doi: 10.1056/ nejm200012073432304

Kimura, A. (2010). Molecular basis of hereditary cardiomyopathy: abnormalities in calcium sensitivity, stretch response, stress response and beyond. J. Hum. Genet. 55, 81-90. doi: 10.1038/jhg.2009.138

Lan, F., Lee, A. S., Liang, P., Sanchez-Freire, V., Nguyen, P. K., Wang, L., et al. (2013). Abnormal calcium handling properties underlie familial hypertrophic cardiomyopathy pathology in patient-specific induced pluripotent stem cells. Cell Stem Cell 12, 101-113. doi: 10.1016/j.stem.2012.10.010

Li, B., Zhan, Y., Liang, Q., Xu, C., Zhou, X., Cai, H., et al. (2021). Isogenic human pluripotent stem cell disease models reveal ABRA deficiency underlies cTnT mutation-induced familial dilated cardiomyopathy. Protein Cell doi: 10.1007/ s13238-021-00843-w [Epub Online ahead of print].

Lian, X., Zhang, J., Azarin, S. M., Zhu, K., Hazeltine, L. B., Bao, X., et al. (2013). Directed cardiomyocyte differentiation from human pluripotent stem cells by modulating Wnt/beta-catenin signaling under fully defined conditions. Nat. Protoc. 8, 162-175. doi: 10.1038/nprot.2012.150

Lombardi, R., Bell, A., Senthil, V., Sidhu, J., Noseda, M., Roberts, R., et al. (2008). Differential interactions of thin filament proteins in two cardiac troponin $\mathrm{T}$ mouse models of hypertrophic and dilated cardiomyopathies. Cardiovasc. Res. 79, 109-117. doi: 10.1093/cvr/cvn078

Molt, S., Bührdel, J. B., Yakovlev, S., Schein, P., Orfanos, Z., Kirfel, G., et al. (2014). Aciculin interacts with filamin $\mathrm{C}$ and Xin and is essential for myofibril assembly, remodeling and maintenance. J. Cell Sci. 127, 3578-3592.

Morimoto, S., Lu, Q. W., Harada, K., Takahashi-Yanaga, F., Minakami, R., Ohta, M., et al. (2002). Ca(2+)-desensitizing effect of a deletion mutation Delta $\mathrm{K} 210$ in cardiac troponin $\mathrm{T}$ that causes familial dilated cardiomyopathy. Proc. Natl. Acad. Sci. U. S. A. 99, 913-918. doi: 10.1073/pnas.02262 8899

Morita, H., Seidman, J., and Seidman, C. E. (2005). Genetic causes of human heart failure. J. Clin. Invest. 115, 518-526. doi: 10.1172/jci24351

Otten, J., Van Der Ven, P. F., Vakeel, P., Eulitz, S., Kirfel, G., Brandau, O., et al. (2010). Complete loss of murine Xin results in a mild cardiac phenotype with altered distribution of intercalated discs. Cardiovasc. Res. 85, 739-750. doi: $10.1093 / \mathrm{cvr} / \mathrm{cvp} 345$

Pacholsky, D., Vakeel, P., Himmel, M., Lowe, T., Stradal, T., Rottner, K., et al. (2004). Xin repeats define a novel actin-binding motif. J. Cell Sci. 117, 52575268. doi: $10.1242 /$ jcs.01406

Sallam, K., Kodo, K., and Wu, J. C. (2014). Modeling Inherited Cardiac Disorders. Circ. J. 78, 784-794.

Sanger, J. W., Wang, J., Fan, Y., White, J., and Sanger, J. M. (2010). Assembly and dynamics of myofibrils. J. Biomed. Biotechnol. 2010:858606.

Savla, J. J., Nelson, B. C., Perry, C. N., and Adler, E. D. (2014). Induced pluripotent stem cells for the study of cardiovascular disease. J. Am. Coll. Cardiol. 64, 512-519.

Sfichi-Duke, L., Garcia-Cazarin, M. L., Sumandea, C. A., Sievert, G. A., Balke, C. W., Zhan, D. Y., et al. (2010). Cardiomyopathy-causing deletion K210 in cardiac troponin $\mathrm{T}$ alters phosphorylation propensity of sarcomeric proteins. J. Mol. Cell. Cardiol. 48, 934-942. doi: 10.1016/j.yjmcc.2010.01.005

Sinn, H. W., Balsamo, J., Lilien, J., and Lin, J. J. (2002). Localization of the novel Xin protein to the adherens junction complex in cardiac and skeletal muscle during development. Dev. Dyn. 225, 1-13. doi: 10.1002/dvdy.10131

Solaro, R. J., and Rarick, H. M. (1998). Troponin and tropomyosin: proteins that switch on and tune in the activity of cardiac myofilaments. Circ. Res. 83, 471-480. doi: 10.1161/01.res.83.5.471

Sun, N., Yazawa, M., Liu, J., Han, L., Sanchez-Freire, V., Abilez, O. J., et al. (2012). Patient-specific induced pluripotent stem cells as a model for familial dilated cardiomyopathy. Sci. Transl. Med. 4:130ra147.

van der Ven, P. F., Ehler, E., Vakeel, P., Eulitz, S., Schenk, J. A., Milting, H., et al. (2006). Unusual splicing events result in distinct Xin isoforms that associate differentially with filamin c and Mena/VASP. Exp. Cell Res. 312, 2154-2167. doi: 10.1016/j.yexcr.2006.03.015

Villard, E., Perret, C., Gary, F., Proust, C., Dilanian, G., Hengstenberg, C., et al. (2011). A genome-wide association study identifies two loci associated with heart failure due to dilated cardiomyopathy. Eur. Heart J. 32, 1065-1076.

Wang, D. Z., Reiter, R. S., Lin, J. L., Wang, Q., Williams, H. S., and Krob, S. L. (1999). Requirement of a novel gene, Xin, in cardiac morphogenesis. Development 126, 1281-1294. doi: 10.1242/dev.126.6.1281

Wang, Q., Lin, J. L., Wu, K. H., Wang, D. Z., Reiter, R. S., Sinn, H. W., et al. (2012). Xin proteins and intercalated disc maturation, signaling and diseases. Front. Biosci. 17:2566-2593. doi: 10.2741/4072

Wang, Q., Lin, J. L.-C., Erives, A. J., Lin, C.-I., and Lin, J. J.-C. (2014). "Chapter Three - New Insights into the Roles of Xin Repeat-Containing Proteins in Cardiac Development, Function, and Disease," in International Review of Cell and Molecular Biology, ed. K. W. Jeon (Cambridge, Massachusetts: Academic Press), 89-128. doi: 10.1016/b978-0-12-800180-6.00003-7

Wang, Q., Lu, T. L., Adams, E., Lin, J. L., and Lin, J. J. (2013). Intercalated disc protein, mXinalpha, suppresses p120-catenin-induced branching phenotype via its interactions with p120-catenin and cortactin. Arch. Biochem. Biophys. 535, 91-100. doi: 10.1016/j.abb.2012.12.018

Wei, B., and Jin, J. P. (2011). Troponin T isoforms and posttranscriptional modifications: evolution, regulation and function. Arch. Biochem. Biophys. 505, 144-154. doi: 10.1016/j.abb.2010.10.013

Conflict of Interest: The authors declare that the research was conducted in the absence of any commercial or financial relationships that could be construed as a potential conflict of interest.

Copyright (c) $2021 \mathrm{Li}$, Guo, Zhan, Zhou, Li, Zhao, Sun, Xu and Liang. This is an open-access article distributed under the terms of the Creative Commons Attribution License (CC BY). The use, distribution or reproduction in other forums is permitted, provided the original author(s) and the copyright owner(s) are credited and that the original publication in this journal is cited, in accordance with accepted academic practice. No use, distribution or reproduction is permitted which does not comply with these terms. 